\title{
Celiac disease and obesity
}

\section{Diagnosed and untreated CD}

According to the Global Burden of Disease Study published in The Lancet medical journal, Kuwait was considered to be the forth country in obesity, where $50 \%$ of women are overweight, and Nearly $70 \%$ of Kuwaiti males over 15 , are overweight or obese. Lifestyle plays a role in this situation, also some diseases like celiac disease, and other digestive problems.

Some studies showed that obesity and overweight celiac diagnosis is largely an adult phenomenon, and also can be in children. If the medical community wants to take obesity seriously, they should consider CD in their plan. Celiac disease is an autoimmune disease which is triggered by gluten, a protein found in wheat, barleyand rye that causes the autoimmune system to fight the presence of gluten in the small intestine. It also can damage the villi's in the small intestine which reflects many related conditions (Figure 1).
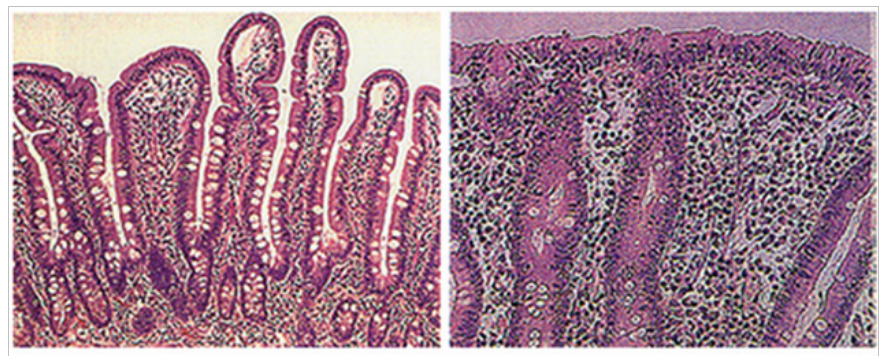

Healthy Villi

Damaged Villi

Figure I Healty Villi vs. Damaged Villi.

Celiac disease's only treatment is to follow a gluten free diet for life. CD is considered by Physicians is mostly in poor BMI people, where the body mass index showed a low number. Physicians were trained at medical school to think that $\mathrm{CD}$ is a rare condition, and it causes weight loss, while celiac disease, according to recentstudy results that it can cause diarrhea and weight loss, between $22-32 \%$,
Volume 4 Issue 2 - 2016

\author{
Suad F AIFuraih \\ Kuwait voluntary celiac awareness team, Kuwait
}

Correspondence: Suad F AlFuraih, Founder of Kuwait voluntary celiac awareness team, Kuwait, Email s.al-feraih@hotmail.com

Received: February 16, 2016 | Published: February 19, 2016

while up to one in three of US adult celiac patients, are overweight or obese.

With a sample size of 258 patients, $C D$ center at Chicago University found that $38 \%$ of the patients were either overweight $(26 \%)$ or obese $(12 \%)$. Over half of the patients $(56.6 \%)$ had a normal BMI, and just $5.4 \%$ of the patients were underweight, a condition that many health care practitioners erroneously consider essential to a celiac diagnosis.

\section{A study that was published by pubmed in} September 2010 concluded that

Obesity is more common in children with $\mathrm{CD}$ than previously recognized. In the appropriate clinical setting, $\mathrm{CD}$ must be considered even in obese children.

Untreated celiac disease symptoms can vary greatly from person to person, it can cause many problems to the patient, migraine, abortion, unexplained anemia, brain and nervous disorders.

Referring also to a study published by Ncpi-Nutrients 2014, 3 out of 17 patients were obese and presented with BMI $\geq 30$ (Figure 2).

Gluten Enteropathy is a common cause of weight issues in populations that consume grains as a diet staple. As a conclusion, consider celiac (coeliac) disease when you study obesity.

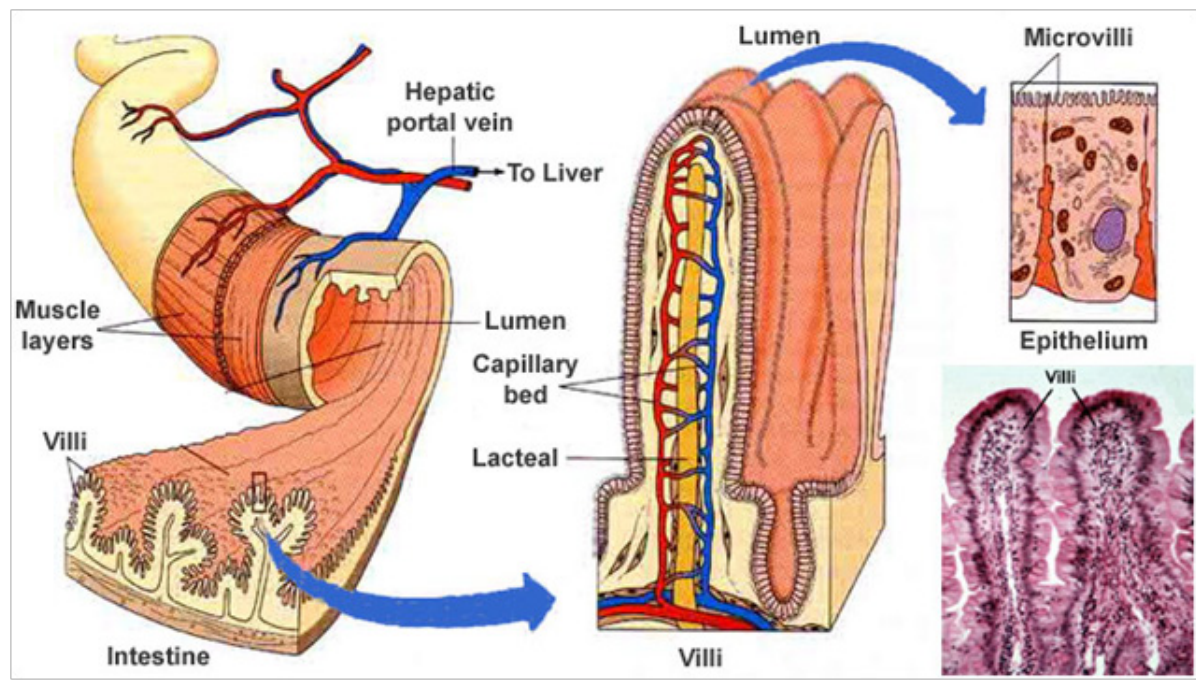

Figure 2 Normal villi. 


\section{Acknowledgements}

None.

\section{Conflict of interest}

The author declares no conflict of interest. 